\title{
Dinámica de la calidad e inequidad del desarrollo humano en la región noreste de México, 1995-2005
} Francisco José Zamudio Sánchez* Alejandro Corona Ambriz ${ }^{* *}$ Yeranui Solorio Elizalde ${ }^{\star * *}$

\section{Resumen}

Se analiza la dinámica del desarrollo humano en el periodo 1995-2005 siguiendo las ideas de Naciones Unidas (NU) para la región noreste de México, constituida por Nuevo León y Tamaulipas. En contraste con lo usual, para los incrementos se propone una tasa de cambio promedio que sea poco afectada por la ley de los rendimientos decrecientes, además los índices usados son invariantes durante el periodo, en oposición a Nu, y se usan dos índices de desarrollo, uno relativo al género, otro de inequidad y uno más de calidad. Ambos estados tienen condiciones de desarrollo superiores a las promedio del país y en equidad de género redujeron notoriamente la desigualdad. A pesar de ello, a escala mundial muestran rezagos importantes. Nuevo León es el más desarrollado pero la educación infantil ha declinado. Tamaulipas muestra superioridad en empleo de recursos. La distribución del desarrollo humano en la región no es equitativa.

Palabras clave: índices de desarrollo humano, inequidad del desarrollo humano, curvas de calidad.

\section{Abstract}

We analyze the dynamics of human development in the period 1995-2005 following the ideas of the United Nations (Nu) for the Northeast region of Mexico, composed of Nuevo Leon and Tamaulipas. In contrast to the usual, for the increments it is proposed an average exchange rate that is not affected by the law of diminishing returns, the indices used are invariant during the period, as opposed to the UN, and we use two indices of development, one related to gender, one to inequity, and one more to quality. Both states have development conditions higher than the national average and gender equity markedly reduced inequality. However worldwide both states show significant lags. Nuevo Leon is the most developed but childhood education has declined. Tamaulipas shows superiority in resource use. The distribution of human development in the region is not fair.

Keywords: human development index, inequality in human development, quality curves.

* Departamento de Estadística, Matemática y Cómputo, División de Ciencias Forestales, Universidad Autónoma Chapingo.Correo electrónico: fjzams@yahoo.com

** Departamento de Estadística, Matemática y Cómputo, División de Ciencias Forestales, Universidad Autónoma Chapingo. Correo electrónico: ambrizcor@yahoo. com.mx

***Egresada de la Universidad Autónoma Chapingo, División de Ciencias Forestales. Correo electrónico: yeranuise@gmail.com 


\section{Introducción}

El desarrollo humano se refiere a la expansión de las capacidades humanas bajo una condición de libertad para elegir lo que se necesite entre diferentes modos de vida, frente a las opciones de que se dispone y para alcanzar los objetivos que satisfagan las expectativas formadas y deseadas durante el desarrollo personal. La perspectiva impulsada por el Programa de las Naciones Unidas para el Desarrollo (PNUD) ${ }^{1}$ reconoce que todos los niveles de desarrollo tienen capacidades y opciones que son esenciales para que las personas participen en las decisiones de una comunidad procurando que se desarrollen plenamente. Las capacidades y opciones esenciales son: mantenerse vivo gozando de salud y de una vida larga; obtener conocimientos; comunicarse libremente; participar en la vida de la comunidad; y contar con los recursos necesarios para llevar una vida digna. No contar con estas capacidades y opciones esenciales origina que otras oportunidades se vuelvan inaccesibles. El PNUD enuncia otras capacidades y opciones que considera relevantes para el desarrollo humano: la libertad política, económica, social y cultural; la disponibilidad de oportunidades con fines productivos o de creación; el respeto por sí mismo; el ejercicio pleno de los derechos humanos y la conciencia de pertenecer a una comunidad. ${ }^{1}$

Amartya Sen, principal representante de la crítica filosófica a la economía del bienestar y la aplicación de la teoría de las capacidades a la medición del desarrollo humano, sostiene que la maximización de la utilidad resulta muy mala descripción del bienestar de las personas que habitan un lugar; un gobierno tiene que ser calificado en función de las capacidades reales de sus ciudadanos, es por ello que en la construcción de los índices se consideran variables que influyen en el desarrollo físico, intelectual y social de los habitantes de una región, estado o municipio; pero aún así ignora muchos aspectos vitales en la toma de decisiones de un individuo,

${ }^{1}$ Programa de las Naciones Unidas para el Desarrollo (PNUD), organización autónoma de la Organización de las Naciones Unidas (ONU), establecida por su Asamblea General en 1965. El PNUD colabora con 150 gobiernos y 30 agencias intergubernamentales y proporciona ayuda técnica con el fin de mejorar el nivel de vida y promover el crecimiento económico en los países subdesarrollados de Asia, África, América Latina, Oriente Próximo y algunas partes de Europa. 
relacionados con preocupaciones sociales, el bienestar de las generaciones futuras o cuestiones éticas.

Existen enfoques que usan otra información, por ejemplo, la utilidad personal que se concentra en los placeres, la felicidad o el deseo de la realización; la opulencia absoluta o relativa, ésta referida a los paquetes de bienes, el ingreso real o la riqueza real; la evaluación de las libertades negativas que se concentra en la ejecución de procesos para que se cumplan los derechos de libertad y las reglas de no interferencia; las comparaciones de los medios de libertad, por ejemplo, la que se refiere a la tenencia de "bienes primarios", como en la teoría de la justicia de Rawls y la comparación de la tenencia de recursos como una base de igualdad justa, como en el criterio de la "igualdad de recursos" de Dworkin (Sen, 1980).

Las teorías de formación del capitalismo sólo ven a las personas como medios y no como fines, cuando se aplica el enfoque sobre la capacidad a la ventaja de una persona, es evaluar en términos de su habilidad real para lograr funcionamientos valiosos como parte de la vida. El enfoque para la evaluación totalizadora de un lugar, así como para la elección de las instituciones y de la política, considera los conjuntos de las capacidades individuales como si constituyeran una parte indispensable y central de la base de información pertinente de tal evaluación (Sen, 1987).

"Es necesario no consumir hoy para tener un mayor consumo mañana" es la base de los modelos formales matemáticos del crecimiento económico; estipulan que el crecimiento del ingreso nacional depende de dos factores: del volumen de ahorros y de la productividad de la inversión efectuada por esos ahorros; es decir, la tasa de ahorro y la razón incremental del capital producto. En modelos más recientes se reconoce que la variable capital producto depende del avance tecnológico, los supuestos del modelo son:

1. El ingreso total depende de dos factores de producción: trabajo y capital.

2. Estos factores son homogéneos internamente, es decir, todas las unidades de capital son sustituibles entre sí y lo mismo pasa para el trabajo.

3. La tecnología es una variable exógena, no es objeto de explicación dentro del modelo, no es dependiente en ningún modelo (Kaldor, 1961). 
Con el peso que tiene la tecnología en el avance económico se tomó conciencia de que la creatividad es un recurso valioso: en la compra de nueva tecnología el dispositivo vale $20 \%$ del precio mientras que el resto paga todo el trabajo de diseño y publicidad del equipo; es decir, la verdadera riqueza de un país no es el capital sino su gente. Un lugar donde los habitantes tienen las condiciones para desarrollarse plenamente, tiende a generar riqueza.

El sureste de Asia y América Latina tenía condiciones similares durante los años de la década de 1960; Asia hizo dos cosas: reforma agraria y escuela universal; tuvo un crecimiento económico superior al $6 \%$ anual, menos del $4 \%$ en desempleo y la productividad de los trabajadores aumentó al 3\% anual; por otro lado, América Latina no instrumentó estas políticas o no las desarrolló de forma adecuada, y tuvo la mitad del crecimiento económico de Asia, el desempleo superó el $10 \%$ y la productividad de sus trabajadores creció menos de $0.5 \%$; no hay una receta estándar para hacer crecer una economía, lo ideal es invertir en sectores que lleven al pleno desarrollo humano, estas estrategias son las que han tomado varios países y se vieron cambios positivos en la economía (Ranis, Stewart y Ramírez, 2000).

El proceso y el bienestar del conjunto de la comunidad resultan, de forma espontánea, de la búsqueda del interés particular. El Estado no ha de interferir si no es para regular la convivencia de interés contrapuesto de forma que no es preciso renunciar al propio interés y al propio beneficio para asegurar los intereses de todos (Sen, 1987).

A principios del siglo xxi es frecuente la expresión 'igualdad de oportunidades' en las políticas sociales de la Comunidad Europea; se refiere a un conjunto de políticas sociales que tienen por objetivo dinamizar el pluralismo junto con el acceso a los puestos y a los ingresos sociales. Entre éstas cabe mencionar las políticas de igualdad de oportunidad entre hombres y mujeres y aquellas que se dirigen a las personas con discapacidad, inmigrantes o a otros sectores de la población que, por razones diversas, encuentran problemas para tener acceso de forma equitativa a diferentes servicios para su pleno desarrollo.

Sen (1994) define pobreza como una privación inaceptable de libertades de los individuos, una condición de vida que limita sus capacidades para vivir la clase de vida en la que los individuos se pueden desarrollar plenamente. El término de pobreza humana distingue esa privación de la 
más estrecha pobreza de ingreso, que es una definición más convencional limitada a la privación de ingresos o consumo. Asimismo, para una vida humana decorosa libre de la miseria propone considerar al menos cinco capacidades básicas:

- la capacidad para vivir libre del hambre,

- la capacidad para vivir una vida libre de enfermedades previsibles y mortalidad prematura,

- la capacidad para vivir libre del analfabetismo literal y numérico,

- la capacidad de acceder a servicios sanitarios básicos (agua potable, eliminación de excretas, electricidad, aseo urbano), y

- la capacidad para obtener empleo.

En este enfoque de desarrollo de capacidades como expresión de opciones para hacerlo se circunscribe la propuesta de desarrollo humano de Sen.

El Informe Mundial sobre Desarrollo Humano fue publicado por primera vez en 1990 con el objetivo principal de establecer a la persona como el centro del proceso de desarrollo en el ámbito del debate económico, político y social (PNUD, 2007-2008). El desarrollo humano se plantea en esencia como un nuevo paradigma analítico y propositivo, a partir del cual se revalora y genera la ampliación de las capacidades de las personas en la vida comunitaria; asimismo, se plantea como una propuesta ética que señala y propone límites diferentes en el proceso histórico de construcción de una nueva sociedad.

Los índices de desarrollo humano son elementos metodológicos que desempeñan una función importante al hacer notar las carencias y privaciones que tienen muchas personas en el mundo, pero, puesto que son el resultado de combinaciones lineales de una o varias variables que influyen en el desarrollo, éstos solamente proporcionan un valor aproximado de la medida de desarrollo que representan, en un país, un estado, una región o un municipio.

En este trabajo se analiza el desarrollo humano de la región noreste de México integrada por Nuevo León y Tamaulipas (Bassols, 1999), mediante el Índice de Desarrollo Humano con Producto Interno Bruto Per Cápita (IDHP); el Índice de Desarrollo Humano con Servicios (IDHS) y el 
Índice de Desarrollo Humano relativo al Género (IDG). Cabe mencionar que el IDHP está compuesto por los siguientes factores: salud, medida por la esperanza de vida al nacer; educación, medida por la alfabetización infantil y de adultos; e ingreso, medido por el producto interno bruto per cápita (PIBP). El IDHS está constituido por los mismos indicadores que el IDHP, a excepción del indicador del ingreso, el cual se sustituye por el de servicios públicos (agua entubada, drenaje y energía eléctrica). El índice de desarrollo humano relativo al género (IDG) se estructura sobre la misma base del IDHP, pero ajusta los resultados para mostrar las diferencias económicas, educativas y de salud entre hombres y mujeres. Los valores de los tres índices mencionados se presentan en la sección de "Análisis de resultados de la región noreste" en las tres primeras subsecciones.

La inequidad de ingresos que se observa en el desarrollo humano de la región de estudio es otra de las preocupaciones que motivan el análisis cuando se refiere al bienestar de las personas. De manera general, la inequidad se define como el tamaño de las diferencias relativas en dotaciones de recursos de unidades que pertenecen a un mismo ambiente particular (Pettinato, 2002:23). Por ello, también se calculó el Índice de Inequidad para el Indice de Desarrollo Humano con Producto Interno Bruto Per Cápita (InQIDHP), cuyos valores para la región aparecen en la subsección "Variación y equidad" de la parte de "Resultados". Además, se construyeron curvas de calidad municipal que permitieron conocer la eficiencia con la que los municipios transforman su ingreso en desarrollo: salud, educación y servicios públicos (agua entubada, electricidad y drenaje). Los resultados aparecen en la parte de "Curvas de calidad".

A partir de un análisis crítico y comparativo entre los resultados obtenidos de los años 1995, 2000 y 2005, y con base en los tres índices antes mencionados (IDHP, IDHS e IDG), podemos analizar los avances o retrocesos de la región, tomando en cuenta que ésta posee una extensión territorial de poco más del $7 \%$ del territorio nacional; está constituida por 94 municipios; tiene 7223530 habitantes (casi 7\% de la población nacional), de los cuales 3639284 son mujeres y 3584246 son hombres; más de 60\% de la población económicamente activa son hombres y dicha región aporta $10.63 \%$ del PIB nacional (INEGI, 2005). Los avances y retrocesos fueron calculados con un promedio de dos tasas de crecimiento, una relativa al avance que se tiene al inicio del periodo bajo análisis y la otra referenciada 
a lo que le falta al indicador o índice respectivo para tener las opciones mínimas que permitan el desarrollo de las capacidades de las personas.

\section{Metodología}

\section{Índices}

El proceso del desarrollo consiste en la eliminación de las principales fuentes de privación de libertad, como la pobreza y la tiranía, la escasez de oportunidades económicas y las privaciones sociales sistemáticas, el abandono en el que pueden encontrarse los servicios públicos y la intolerancia o el exceso de intervención de los Estados respectivos (PNUD, 2005). Para la medición del desarrollo, en la actualidad se tiene preferencia por el uso de un índice y es el enfoque del trabajo.

En los tres índices que se construyen: IDHP (PNUD, 1990), IDG (PNUD, 1995) e IDHS (Ramírez, 1999) se calculan varios indicadores. Tanto indicadores como índices fueron construidos de acuerdo con la metodología del PNUD. La excepción es el IDHs, ya que este indicador no lo proporciona este organismo, pero se siguió un criterio análogo al usado para los otros índices (Zamudio, Corona y González, 2007). Para los informes de México sobre desarrollo humano 1995, 2000 y 2005 se construyeron los indicadores de cada variable de la siguiente manera:

$$
\text { In }=\frac{\text { valor }- \text { min }}{\text { máx }- \text { min }}
$$

Donde:

Valor: es aquel que toma la variable correspondiente.

Mín: es el valor mínimo que toma la variable correspondiente.

Máx: es el valor máximo que toma la variable correspondiente.

Cuando la variable es el producto interno bruto per cápita, antes de usar la fórmula se transforma el valor usando la función logaritmo natural.

La escala básica de este estudio es la municipal, así, de los 94 municipios de la región se obtendrá un indicador por cada variable considerada. 


\section{Índice de Desarrollo Humano con Producto Interno Bruto Per Cápita (idhp)}

El costo de la canasta básica varía en cada lugar, por ello revisaremos el IDHP porque considera el producto interno bruto per cápita en dólares -Paridad de Poder Adquisitivo (PPA)—. Este índice representa aquella parte del desarrollo humano que no está considerada en la salud ni en la educación.

La fórmula empleada asigna el mismo peso a la educación, la salud y el ingreso. Se utilizan cuatro variables: esperanza de vida, tasa de alfabetización, tasa de niños de seis a 14 años que saben leer y escribir (matrícula infantil) y producto interno bruto per cápita a pesos constantes de 1993. El IDHP se calculó con la fórmula:

$$
i d h p=\frac{\operatorname{InEsp}+\left(\frac{2}{3} \operatorname{In} A l f+\frac{1}{3} \operatorname{InMat}\right)+\operatorname{InPibp}}{3}
$$

Donde:

InEsp: indicador de esperanza de vida.

InAlf: indicador de alfabetización.

InMat: indicador de matriculación infantil.

InPibp: indicador del producto interno bruto per cápita.

Los máximos y mínimos para cada variable involucrada en los cálculos de los índicadores del IDHP se presentan en el cuadro $1 .^{2}$

\section{Índice de Desarrollo Humano con Servicios (idhs)}

Este índice considera los servicios públicos básicos como son agua entubada, drenaje y electricidad en sustitución del PIBP.

Se utilizan las siguientes seis variables: esperanza de vida, tasa de alfabetización, tasa de niños de seis a 14 años que saben leer y escribir, tasa

${ }^{2}$ Para corroborar la manera en que se obtuvo la esperanza de vida, tasa de matriculación infantil, tasa de alfabetización y el PIBP de cada uno de los municipios de los estados de la República Mexicana para los años 1995, 2000 y 2005, consultar la memoria técnica en la página de la red: http//www.chapingo.mx/dicifo/demyc/idh. 


\section{Cuadro 1. Máximos y mínimos de las variables usadas en los índices de desarrollo}

\begin{tabular}{|l|c|c|}
\hline \multicolumn{1}{|c|}{ Variable } & Máximo & Mínimo \\
\hline Esperanza de vida (años) & 90 & 30 \\
Tasa de alfabetización (población mayor de 14 años) & 100 & 0 \\
Tasa de alfabetización de niños de 6 a 14 años & 100 & 0 \\
PIB per cápita (dólares PPA) ${ }^{[1]}$ & 40,000 & 100 \\
Tasa de habitantes con drenaje & 100 & 0 \\
Tasa de habitantes con agua entubada & 100 & 0 \\
Tasa de habitantes con electricidad & 100 & 0 \\
\hline
\end{tabular}

[1] Las tasas PPA permiten determinar el número de unidades de la moneda de un país necesarias para adquirir la misma canasta representativa de bienes y servicios que un dólar de Estados Unidos adquiriría en tal país.

Fuente: Departamento de Estadística, Matemática y Computo (DEмyc).

de habitantes con drenaje, tasa de habitantes con agua entubada y tasa de habitantes con electricidad. El IDHS se calculó de la siguiente manera:

$$
i d h s=\frac{\operatorname{InEsp}+((2 / 3) \operatorname{In} A l f+(1 / 3) \operatorname{InMat})+((1 / 3) \operatorname{InDre}+(1 / 3) \operatorname{In} A g u+(1 / 3) \operatorname{InEle})}{3}
$$

Donde:

InEsp, InAlf e InMat son como antes.

InDre: indicador de drenaje.

InAgu: indicador de agua.

InEle: indicador de electricidad.

Este índice es congruente con la idea de Naciones Unidas sobre desarrollo, la cual asigna el mismo peso a la salud, la educación y la componente que representa el resto del bienestar que no está en estas dos. Los tres servicios se ponderan de igual manera ya que se determinó que tienen la misma importancia (DEMyc, 2007). 


\section{Índice de Desarrollo Humano relativo al Género (idg)}

El Índice de Desarrollo Humano relativo al Género mide el grado de adelanto en las mismas capacidades básicas que el IDHP, pero incorpora la desigualdad entre hombres y mujeres en cuanto al grado de dicho adelanto. Se impone una penalidad a la desigualdad, así el valor del IDG será menor al IDHP a medida que haya una mayor diferencia entre el nivel de adelanto global de mujeres y hombres.

$$
i d h p=\frac{\operatorname{InEsp_{id}}+\operatorname{InE} d u_{i l}+\operatorname{InPibp}_{i l}}{3}
$$

Donde:

InEsp $p_{i d}$ : índice de esperanza de vida igualmente distribuido.

InEd $u_{i d}$ : índice de educación igualmente distribuido.

InPibp $_{i d}$ : índice del producto interno bruto per cápita igualmente distribuido.

A pesar de seguir conceptualmente la metodología del PNUD, consideramos inadecuado apegarnos a todos los cambios que sugieren a través del tiempo por las razones siguientes.

En primer lugar, todas las modificaciones incorporadas a los índices desde el nacimiento de los informes mundiales en 1990 harían imposible la realización de un análisis longitudinal, propósito de este trabajo. Actualizar para todas las bases de datos los índices a través del tiempo, cada vez que el PNud realiza un cambio, haría una labor anual muy pesada, pero aún menos pertinente por cualquiera de las dos razones siguientes: 1) si los cambios son sustantivos y los índices se modifican drásticamente, entonces cada análisis arrojaría hallazgos diferentes, ocasionando una confusión permanente sobre la evolución del desarrollo y provocando un resultado contrario al que se busca de explicar en tal evolución; 2) si los cambios son mínimos o no cambian el orden del índice, entonces no habría mucho caso en apegarse a la modificaciones sugeridas, pues finalmente lo que proporcionan los índices es una escala ordinal para jerarquizar a las diferentes entidades que se miden.

En segundo lugar, hay cambios cuya justificación no es del todo convincente. En un boletín de 2012, el PNUD en México (PNUD, 2012) da a 
conocer una nueva forma de agregación de los índices: se abandona la media aritmética y se adopta la media geométrica, argumentando el carácter sustituto que tiene la primera de los índices que la componen, situación de la que no escapa la segunda, porque si dos índices componentes intercambian valores, permaneciendo el tercero constante, la media geométrica permanece invariante; así, de los dos componentes que intercambian valor, uno de ellos sustituye al otro. Por otro lado, en una media geométrica, será el valor más pequeño, de los valores que intervienen, el que tendrá mayor importancia en el valor de la media geométrica: por ejemplo, $(0.69104 \times 0.68597 \times 0.07027)^{1 / 3}=0.32175$, donde el valor 0.07027 está más cerca de 0.32175 que los otros dos, mostrando su relevancia en el valor de la media geométrica, mientras que la media aritmética, 0.53726, está más cerca de los dos primeros que del último. De esta manera, en la media geométrica, la consideración de dar el mismo peso a las tres dimensiones del desarrollo se pierde y surge la pregunta, ¿qué es más crítico, lo indeseable que tiene el carácter sustitutivo o considerar con mayor peso una dimensión del desarrollo humano en relación con las otras dos? ¿Acaso no darle mayor peso a una de las dimensiones es equivalente a sustituir la importancia de las otras? Si los pesos a las dimensiones no son importantes y se trata de señalar la dimensión más crítica del desarrollo humano, entonces el índice que le da todo el peso a una sola dimensión es el mínimo de las tres dimensiones, el cual mediría el desarrollo de un territorio con la dimensión que tiene las menores opciones para el mismo. Tal selección de medida reduciría el ya bastante reducido número de componentes usadas en la construcción del índice, además induciría otro problema en la comparación de las entidades medidas: transversalmente no se podrían comparar porque unas mediciones estarían referidas a una dimensión y otras a otra.

En tercer lugar, la medición del InEsp del PNUD sufrió, aparentemente, ligeros cambios con el informe referente a 2010. De acuerdo con los umbrales establecidos antes de la medición de 2010, se esperaba que una persona en condiciones mínimas de salud lograra vivir 85 años. Por el contrario, las personas en las condiciones más precarias deberían prolongar su vida al menos 25 años. Para la medición de 2010 ambas cotas se redujeron; ahora se espera que las personas con condiciones básicas de salud vivan menos años (83.4) y las personas en condiciones precarias también (20) 
(PNUD, 2011). El cambio de las cotas superior e inferior, en 2010, ocasionará que los nuevos índices sean mayores que los calculados antes para una misma esperanza de vida. Hacer que mejore el indicador de la esperanza de vida cambiando los umbrales que se tenían en mediciones pasadas, es una acción que no tiene que ver con el desarrollo humano y exhibe poca seriedad del PNUD. Si, de otro modo, el PNUD considera que la edad máxima en condiciones mínimas de salud ahora se redujo 1.6 años, es un cambio, por precisión en términos de esperanza de vida, difícil de justificar y que sólo obedece a una convención aproximada por conocimiento empírico; entonces ¿̇por qué no 85 años que es el umbral usado desde 1990? El efecto final en México es que los índices de esperanza de vida de los estados se vieron favorecidos en el quinquenio 2005-2010, precisamente cuando varios estados de la república disminuyeron su esperanza de vida por el alarmante ascenso del número de homicidios (Durango y Chihuahua entre los más afectados, pero también otros 13 estados). ${ }^{3}$

El cuadro 2 muestra un comparativo entre los resultados de la medición pasada y vigente del InEsp. El método de cálculo vigente induce un

Cuadro 2. Comparativo entre los resultados de la medición
pasada y vigente del InEsp

\begin{tabular}{|c|c|c|}
\hline \multirow{2}{*}{$\begin{array}{c}\text { Esperanza de vida } \\
\text { (años) }\end{array}$} & Medición anterior a 2010 & Medición de 2010 \\
\cline { 2 - 3 } & 0.75000 & 0.78864 \\
\hline 70 & 0.76667 & 0.80442 \\
\hline 71 & 0.78333 & 0.82019 \\
\hline 72 & 0.80000 & 0.83596 \\
\hline 73 & 0.81667 & 0.85174 \\
\hline 74 & 0.83333 & 0.86751 \\
\hline 75 & \multicolumn{2}{|c|}{} \\
\hline
\end{tabular}

Fuente: Elaboración propia.

${ }^{3}$ Para un informe exhaustivo de la esperanza de vida, consultar el informe estadístico sobre desarrollo humano en México, 1995-2010, en la dirección electrónica: http:// www.chapingo.mx/dicifo/demyc/idh/new/bases_new/?mod=publicaciones\&doc $=$ infor me2010 
efecto de crecimiento en el índice y por tanto en las conclusiones que se hagan. Por ejemplo, en el caso de 71 años, el InEsp es menor a 0.8 y por tanto sería clasificado como de nivel medio, sin embargo, utilizando el método vigente, el índice es catalogado como de nivel alto.

En cuanto al Índice de Desarrollo Humano relativo al Género, el nuevo índice propuesto por el PNUD no considera la dimensión de salud para los hombres (PNUD, 2011; memoria técnica), induciendo con ello un sesgo en la comparación de las condiciones en las que se encuentran éstos respecto de las mujeres. Si se agrega que los hombres viven menos que las mujeres, que la esperanza de vida al nacer representa la medida integrada más fiel de la salud y que la dimensión salud es central para el desarrollo de capacidades de las personas, es inadecuado aceptar un índice de este tipo cuando el propósito es comparar las condiciones de hombres y mujeres, excepto que con ello se pretenda algebraicamente exagerar las desigualdades de género que se tienen. Cualesquiera que sean las razones, consideramos indispensable que la dimensión salud sea incorporada en las comparaciones de género y por ello optamos por ceñirnos al índice que se usaba anteriormente.

Las tres primeras observaciones anteriores explican el porqué no resulta pertinente incorporar los cambios que constantemente propone la oficina del PNUD en un análisis longitudinal del desarrollo humano; y en cuanto al género, la ausencia de la dimensión salud en los hombres presenta al índice antes usado como mejor opción de medición.

\section{Medición del crecimiento de indicadores e índices}

Para el cálculo de los incrementos logrados en el periodo 2000-2005 por cada municipio en el IDHP, IDHS y los indicadores componentes de éstos, se utilizó la expresión siguiente:

$$
\text { Incremento del indice }=\frac{1}{2}\left[\frac{\text { indice } 2005-\text { indice } 2000}{\text { indice } 2000}+\frac{\text { indice } 2005-\text { indice } 2000}{1-\text { indice } 2000}\right]
$$

Para el periodo 1995-2000 se hacen los cambios respectivos. Se trata de promediar el incremento con respecto del logro que se tiene al inicio 
y lo que faltaba para llegar al valor máximo posible de 1, buscando que este valor no sea muy afectado por los rendimientos decrecientes que son propios de todo proceso acumulativo como lo es el desarrollo humano.

Para medir el adelanto hacia la equidad de género, en los años 1995, 2000 y 2005, se usó la siguiente tasa:

$$
\text { Diferencia porcentual }=\frac{i d h p-i d g}{i d h p} * 100
$$

\section{Variación e inequidad}

El Índice de Gini normalmente se utiliza para medir la desigualdad en los ingresos, pero puede utilizarse también para medir cualquier forma de distribución desigual. Es un número entre 0 y 1 , en donde cerca de 0 indica una equidad en la distribución y cerca de 1 señala una concentración de la variable bajo estudio (Martínez, 2001). Se utilizó la siguiente expresión:

$$
\operatorname{In} G=\frac{1}{10000}\left[\sum_{i=1}^{9}\left(X_{i} Y_{1+i} \quad X_{1+i} Y_{i}\right)\right]
$$

Donde:

$Y_{i}$ : porcentaje acumulado de la población hasta el $i$-ésimo decil, y

$X_{i}$ : porcentaje acumulado de la variable o índice estimado para el $i$ ésimo decil de la población.

Para realizar el análisis se define el Índice de Inequidad (InQ) como uno menos el Índice de Gini aplicado al IDHP, el cual se denotará por InQIDHP. La razón de lo anterior obedece a que entre mayor esté concentrada la medida de desarrollo humano entre la población, la condición será más favorable para ella.

Si un estado tiene una distribución inequitativa de las oportunidades para disponer de buenas condiciones de salud, educación o servicios, la oportunidad de desarrollo humano tendrá una distribución desigual entre los municipios. De ahí la importancia de medir la inequidad en la distribución del desarrollo a través del InQIDHP (DEMyc, 2007). 


\section{Calidad municipal}

Las curvas de calidad municipal se utilizan para clasificar a los municipios según la eficiencia de éstos en el empleo del ingreso (PIBP en dólares PPA) para mejorar la salud, la educación y los servicios públicos (agua entubada, drenaje y electricidad), que se consideran esenciales para un buen desarrollo humano.

En breve, decimos que un municipio tiene una mejor calidad que otro si ocurre que ambos tienen el mismo PIBP pero el primero tiene un IDHS mayor que el segundo. Dependiendo de la diferencia que haya en el IDHs será la diferencia en calidad de los municipios. ${ }^{4}$ Se determinan 30 calidades diferentes, donde la 1 representa la mejor calidad porque, a un mismo ingreso, indicaría que en ese territorio es donde más opciones de desarrollo se han ofrecido a los habitantes y la 30 sería donde menos opciones habrían tenido.

\section{Análisis de resultados de la región noreste}

La geografía, como ciencia de estudio de las realidades físicas, humanas y económicas del mundo actual en su proyección histórica de perenne cambio, que se proyecta en forma de sistemas regionales de fenómenos, puede dar poderosas armas en el análisis de las causas, los marcos y las relaciones entre hechos y ayudar a explicar el desequilibrio regional en el México de hoy (Bassols, 1967).

Siguiendo las ideas de Bassols, la diferencia en el desarrollo de Tamaulipas y Nuevo León inicia en la primera mitad del siglo xix en Monterrey. Uno de los impulsos recibidos provino del comercio (y el contrabando) con Estados Unidos. La acumulación de capital se aceleró de manera notable bajo el régimen porfirista, gracias a varios factores, entre ellos, la construcción de los ferrocarriles. Monterrey se convirtió en un gran cen-

${ }^{4}$ La metodología detallada del cálculo de los índices (IDHP, IDHS e IDG), así como las curvas de calidad municipal, y el Índice de Inequidad se encuentran en www.chapingo. $\mathrm{mx} /$ dicifo/demyc/idh. 
tro manufacturero durante el largo periodo de la dictadura, con su política de favorecer la concentración del ingreso, en un principio con capital extranjero predominante.

El espacio comercial del que es centro Monterrey abarca todo Nuevo León y buena parte de Tamaulipas, y sus brazos financieros llegan hasta México y Guadalajara, pero se concentra en el noreste propiamente y el sureste de Coahuila de los cuales obtiene sus materias primas y energía, con importancia minoritaria también de Colima, Nayarit y Veracruz.

Sobre Monterrey también gravitan las ciudades fronterizas más cercanas debido a que es la única ciudad que cuenta con diversos servicios especializados en toda la región noreste del país.

Tamaulipas fue sometida y colonizada por los españoles y hasta el siglo XVIII éstos establecieron misiones franciscanas y dominicas que tuvieron fundamentalmente una base agrícola y ganadera; durante el siglo XIx Tampico y Altamira se volvieron puertas para el comercio exterior de Nuevo León y el resto de México.

Tamaulipas basó sus relaciones comerciales en recursos energéticos, petróleo de Ciudad Madero y gas proveniente de Reynosa, principalmente; en la actualidad esto no resulta decisivo, ya que tiene centrales termoeléctricas mixtas, produce el triple de la electricidad que consume el estado y genera la tercera parte del gas natural extraído en el país.

Esta diferenciación, surgida desde el siglo xix, en los roles de Nuevo León y Tamaulipas en la región, han determinado el estado actual de las opciones que ellos ofrecen a sus habitantes para el desarrollo de sus capacidades.

\section{Índice de Desarrollo Humano con Producto Interno Bruto (idhp)}

Para el análisis de este índice se utilizará el criterio del PNud que establece que un índice es bajo cuando su valor es menor a 0.5 ; medio cuando su valor es mayor o igual a 0.5 pero menor a 0.8 , y alto cuando su valor es mayor o igual a 0.8 y menor o igual a 1 . Cuando un municipio presenta un índice medio o bajo significa que el lugar no cuenta ni con lo mínimo que debería de disponer para satisfacer sus necesidades. 
A escala mundial, en el Informe sobre Desarrollo Humano del año 2005, Islandia (0.968) tiene el mejor IDHP reportado; en este país, la esperanza de vida está alrededor de los 81 años, menos del $1 \%$ de su población es analfabeta y tiene un ingreso alto (36 510 dólares PPA); el IDHP más bajo lo posee Sierra Leona (0.336), donde la esperanza de vida no rebasa los 42 años, $65.2 \%$ de la población es analfabeta y el ingreso es de 806 dólares PPA, que aunque es bajo, está por encima de otros (PNUD, 2007-2008). En ese informe México ocupa la posición 52 de los 177 países considerados, ubicado entre Cuba y Bulgaria. De acuerdo con nuestros cálculos, en el país la esperanza de vida es de 74.61 años, $8.4 \%$ de la población es analfabeta y el ingreso es de 8998.34 dólares Ppa. Es claro que las oportunidades que se tienen en México comparativamente con Islandia tienen un rezago significativo; aspirar a una esperanza de vida alrededor de los 81 años resulta una meta alcanzable para el año 2070 y tener una tasa de analfabetas menor al 1\% tomaría al menos 45 años, en ambos de acuerdo a como estos indicadores han cambiado en los últimos 20 años. Respecto de tener un ingreso en el orden de los 35500 dólares PPA, es una inferencia más compleja por la variabilidad que este indicador presenta, sin embargo, nótese que es en el que mayor rezago se tiene. Así, los territorios en México que se muestran con un Índice de Desarrollo Humano promedio deben encuadrarse en la posición que tiene el país respecto de los países de alto desarrollo y aquellos que se localicen por abajo deben considerarse con rezagos más críticos que los enunciados.

Nuevo León (NLN) ha conservado su alto nivel de desarrollo humano; de 1995 a 2005 creció 8\%, el avance promedio nacional fue de $8.12 \%$ y ocupa el segundo lugar estatal por el IDHP; el Distrito Federal tiene el primer lugar y en último está el estado de Chiapas. El crecimiento reportado por estas entidades es de $8.4 \%$ y $7.99 \%$ respectivamente. El IDHP alcanzado por Nuevo León es un poco más alto que el reportado por Uruguay, Croacia y Costa Rica, mismos que ocupan las posiciones 46, 47 y 48, respectivamente, entre los países considerados por el informe arriba mencionado (PNUD, 2007-2008).

En el año 2000, el estado de Tamaulipas (TAM) pasó de una categoría media a alta; durante los dos quinquenios de análisis el incremento en este indicador fue de $8.81 \%$, ubicándose en la séptima posición nacional. El desarrollo alcanzado por este estado está debajo del reportado por Bulgaria entre los países considerados en el informe. 
En 1995, 34 de los 51 municipios de Nuevo León estaban clasificados con alto desarrollo, y abarcaban $93.14 \%$ de la población. En 2005, esa cantidad aumentó a 48 municipios representando $99 \%$ de la población. Cabe mencionar que en esta región no hay municipios con bajo nivel de desarrollo.

En 1995, de los 43 municipios que conforman el estado de Tamaulipas, 40 tenían un IDHP medio, y habitaba 72.54\% de la población estatal. En 2005, los municipios con nivel alto de desarrollo eran 23 y contenían $91.04 \%$ de los habitantes, un cambio significativo para este estado (cuadro 3).

Cuadro 3. Valores del idhp y sus componentes para el país y los estados de la región noreste, 1995-2000-2005

\begin{tabular}{|c|c|c|c|c|c|c|c|}
\hline & Años & $\begin{array}{c}\text { Esperanza } \\
\text { de vida* }\end{array}$ & InMat & InAlf & $\begin{array}{c}\text { Pib per } \\
\text { cápita** }\end{array}$ & IDHP & $\begin{array}{c}\text { Avance } \\
\text { IDHP (\%) }\end{array}$ \\
\hline \multirow{4}{*}{ Nacional } & 1995 & 72.58 & 0.8599 & 0.8926 & $7,441.90$ & 0.7702 & \\
\cline { 2 - 8 } & 2000 & 73.88 & 0.8728 & 0.9045 & $9,067.94$ & 0.7925 & 6.30 \\
\cline { 2 - 8 } & 2005 & 74.61 & 0.8779 & 0.9146 & $8,998.34$ & 0.7990 & 1.97 \\
\hline \multirow{3}{*}{ Nuevo León } & 1995 & 74.30 & 0.9313 & 0.9606 & $12,343.15$ & 0.8310 & \\
\cline { 2 - 8 } & 2000 & 75.19 & 0.9270 & 0.9658 & $15,897.69$ & 0.8507 & 7.01 \\
\cline { 2 - 8 } & 2005 & 75.87 & 0.9022 & 0.9686 & $16,220.10$ & 0.8535 & 1.01 \\
\hline \multirow{3}{*}{ Tamaulipas } & 1995 & 74.00 & 0.8907 & 0.9388 & $7,768.94$ & 0.7942 & \\
\cline { 2 - 8 } & 2000 & 75.02 & 0.8940 & 0.9480 & $9,751.99$ & 0.8149 & 6.43 \\
\cline { 2 - 8 } & 2005 & 75.73 & 0.9011 & 0.9529 & $10,139.65$ & 0.8229 & 2.67 \\
\hline
\end{tabular}

* Años.

** Dólares PPA.

Fuente: Cálculos propios con datos del INEGI, II Conteo 2005, XII Censo 2000 y I Conteo 1995, XI Censo 1990, Banco de Información Económica (вIE) y Sistema Municipal de Base de Datos (Simbad).

El municipio con el IDHP más alto en la región durante el 2005 fue San Pedro Garza García (0.8823), seguido por San Nicolás de los Garzas (0.8697), Guadalupe (0.8593), Apodaca (0.8592), Santa Catarina (0.8530) y Monterrey (0.8495), todos pertenecientes a Nuevo León. San Pedro Gar- 
za García ocupa el tercer lugar entre los 2454 municipios que conforman la República Mexicana, supera a Malta (0.8780), localizado en la posición 34, y está por debajo de Portugal (0.8970), en la posición 29 del Informe sobre el Desarrollo Humano (PNud, 2007-2008).

Por otra parte, el municipio con menor índice de desarrollo fue Bustamante, NLn (0.7533), seguido por Michihuana, TAM (0.7701), San Nicolás, TAM (0.7702), Mier y Noriega, TAM (0.7760), Burgos, TAM (0.7821) y San Carlos, TAM (0.7851). Bustamante tiene la posición 1051 en la clasificación municipal del país; su desarrollo es superior al reportado por Jamaica (0.7360), con la posición 101, e inferior a Georgia (0.7540), que ocupa el lugar 96 respecto de la clasificación mundial.

Durante la década, en el comportamiento de los componentes del IDHP de la región, sobresale el incremento en el InAlf, el cual registra un avance de $10.56 \%$ en Nuevo León, obteniendo el segundo lugar nacional; Tamaulipas creció $12.37 \%$, ubicándose en el noveno lugar nacional. El municipio con mejor índice en la región es San Nicolás de los Garza (0.9853) y el peor es Bustamante (0.7375), ambos pertenecientes al estado de Nuevo León. El Distrito Federal (0.9805) tiene el primer lugar estatal en alfabetización y el último en la escala nacional es Chiapas (0.7856). Respecto del InPIвP, el primer lugar estatal lo ocupa el Distrito Federal, seguido por Nuevo León que creció $14.45 \%$; Tamaulipas creció $6.22 \%$ y se ubica en la décimosegunda posición, y el último lugar es Oaxaca. El municipio de la región con mayor aporte al ingreso es San Pedro Garza García, NLn (20 377.09 dólares PPA), y los de menor son San Carlos, TAM (4 736.35 dólares PPA), Los Aldamas, NLn (4 242.19 dólares PPA) y San Nicolás, тАм (4 230.91 dólares PPA). En el InEsp, Nuevo León creció 6.78\%, lo cual significa un año 174 días más de vida; pasó de 74.3 a 75.8 años, ubicándose así en el sexto lugar nacional; mientras Tamaulipas reporta un incremento de 7.41\%; es decir, un año 226 días más de vida: este estado pasó de 74 a 75.74 años, ubicándose en el noveno lugar nacional. Para la región, el municipio con mayor esperanza de vida fue Tula, TAM, con 79.93 años, y el valor más bajo lo presentó Camargo, TAм, con 73.11 años. El estado con mayor esperanza de vida es Guerrero, superando a los estados de la región en estudio por casi dos años; el estado con menor esperanza de vida es Chihuahua, con más de tres años de diferencia respecto de la región noreste. Respecto del InMat, el Estado de México 
tiene el primer lugar nacional, Nuevo León ocupa el séptimo (decreció $22.66 \%$ ), Tamaulipas (creció $5.37 \%$ ) se ubica en el octavo lugar y el último es Chiapas. Toda la región noreste se encuentra con un alto desarrollo en este indicador.

En el Informe de Desarrollo Humano 2005 (PNUd, 2007-2008), Japón tiene la mejor esperanza de vida (82 años), la más corta está alrededor de los 40 años en varios países del continente africano; el InEdu más bajo está alrededor de $20 \%$ de población alfabeta y los más altos cercanos a $100 \%$; el ingreso de Luxemburgo (60 228 dólares PPA) es el mayor en esta lista y el menor es Malawi (667 dólares PPA). Respecto de estos extremos, Nuevo León y Tamaulipas se ubican más cerca de los superiores en esperanza de vida y educación pero no así en ingreso, indicando que las opciones posibles con el mismo para el desarrollo son limitadas, aunque no tan críticas como las de los países africanos. Unos cuantos indicadores no pueden medir un proceso pluridimensional como es el caso de los que componen el IDHP que se integran en el mismo con pesos iguales; sin embargo, son referentes que ordenan opciones y capacidades con las que cuentan las personas contrastando situaciones y señalando los aspectos críticos a corregir. La región noreste en general cuenta con las condiciones mínimas para el desarrollo de sus habitantes, el retroceso de Nuevo León en InMat (proxy de la alfabetización infantil) puede ser grave y debe ser atendido.

\section{Índice de Desarrollo Humano modificado con servicios}

En lo que se refiere a servicios, la región noreste ha mantenido un alto nivel de desarrollo. El primer lugar nacional es Aguascalientes (0.8914), seguido por Nuevo León; Tamaulipas tiene el duodécimo lugar y en último lugar encontramos a Oaxaca (0.7666). El municipio con el mejor IDHs de la región es Melchor Ocampo, NLN (0.9153), que ocupa el segundo lugar dentro de los municipios del país; los más bajos son Casas, тAм (0.7392), Bustamante, TAM (0.7225), y San Nicolás, TAм 0.6981). El incremento reportado por Tamaulipas en este índice, durante 1995-2005, es poco más del doble que el presentado en Nuevo León.

En 1995 y respecto del IDHs, Nuevo León tenía 40 municipios clasificados con alto desarrollo, los cuales concentraban $95.1 \%$ de la población 
estatal; en el 2000 creció 2\%, y para el 2005 aumentó a 47 municipios, habitados por $98.6 \%$ de la población estatal. Por otra parte, en 1995 Tamaulipas tenía 16 municipios con alto desarrollo, concentrando $80.3 \%$ de lo población estatal; en el 2000, eran 19 municipios con $87.9 \%$ de la población y para el 2005, 30 municipios, habitados por $97.7 \%$ de la población (cuadro 4).

El componente con mayor avance es el InDre. En Nuevo León el incremento fue de $32.25 \%$, ocupando el quinto lugar nacional; Tamaulipas tuvo un avance de $36.84 \%$, ubicándose en el vigésimo segundo lugar. El municipio con mejor InDre es San Nicolás de los Garza, NLn (0.9938), que ocupa el tercer lugar entre los municipios que conforman el país, y el de más bajo índice es San Nicolás, TAM, al no contar con este servicio. En Tamaulipas, el InEle creció 30.44\%, ubicándose así en la posición 21; Nuevo León avanzó $2.84 \%$ y ocupó el sexto lugar. Mier, TAM (0.9924), es el mu-

\section{Cuadro 4. Valores del idhs y sus componentes para el país y los estados de la región noreste, 1995-2000-2005*}

\begin{tabular}{|c|c|c|c|c|c|c|}
\hline & Años & InAgu & InEle & InDre & IDHS & $\begin{array}{c}\text { Avance } \\
\text { IDHS (\%) }\end{array}$ \\
\hline \multirow{3}{*}{ Nacional } & 1995 & 0.8458 & 0.9280 & 0.7240 & 0.8080 & \\
\cline { 2 - 7 } & 2000 & 0.8743 & 0.9480 & 0.7618 & 0.8293 & 6.87 \\
\cline { 2 - 7 } & 2005 & 0.8902 & 0.9645 & 0.8545 & 0.8497 & 7.21 \\
\hline \multirow{3}{*}{$\begin{array}{c}\text { Nuevo } \\
\text { León }\end{array}$} & 1995 & 0.9448 & 0.9802 & 0.8862 & 0.8754 & \\
\cline { 2 - 7 } & 2000 & 0.9564 & 0.9865 & 0.9105 & 0.8857 & 4.72 \\
\hline \multirow{3}{*}{ Tamaulipas } & 2005 & 0.9545 & 0.9813 & 0.9513 & 0.8911 & 2.67 \\
\cline { 2 - 7 } & 1995 & 0.8888 & 0.9067 & 0.6561 & 0.8244 & \\
\cline { 2 - 7 } & 2005 & 0.9113 & 0.9473 & 0.7336 & 0.8515 & 9.34 \\
\hline
\end{tabular}

* En este cuadro se omiten los valores de esperanza de vida, InAlf e InMat, puesto que ya aparecen en el cuadro anterior.

Fuente: Cálculos propios con datos del INEGI, II Conteo 2005, XII Censo 2000 y I Conteo 1995, XI Censo 1990, Banco de Información Económica (BIE) y Sistema Municipal de Base de Datos (Simbad). 
nicipio con el mejor InEle en la región y el peor es Mendez, TAM (0.8019). El Distrito Federal tiene el primer lugar en ambos indicadores y Oaxaca reporta los valores más bajos, igualmente para ambos indicadores. En Nuevo León el InAgu aumentó 9.29\%, posicionándose en la séptima posición dentro de la escala nacional; Tamaulipas reportó un incremento de $30.44 \%$ en este índice, ocupando el noveno lugar. Apodaca, NLN (0.9884), presentó el mejor InAgu en la región mientras que Casa, TAM (0.4909), y San Nicolás, TAm (0.2912), reportan el más bajo. Dentro de la escala nacional, Aguascalientes (0.9770) tiene el primer lugar y Guerrero (0.6886) el último. Durante la década estudiada son muy notables los avances en los servicios, tanto de ambos estados como en el país.

\section{Índice de Desarrollo Humano relativo al Género}

La discriminación de género es una de las versiones más crudas de la inequidad, el feminismo es una actitud política, una reflexión sobre la experiencia de ser mujer en una sociedad desigual y la posibilidad de lograr que esta decisión cuente en la vida pública, es decir, lo personal es político (Jagaar, 1983). El machismo define el modo de vida en las sociedades; la desigualdad en el interior del hogar por reglas ancestrales somete a las mujeres y las hace víctimas de violencia doméstica, desigualdad en la libertad sexual, desigualdad en la oportunidad educativa, etcétera (McKinnon, 1987:36); así, tan sólo dos de cada tres analfabetas en el mundo son mujeres, quienes sufren además desigualdad laboral, pues están típicamente asignadas a puestos de menor calidad y valoración social, constituyendo $70 \%$ de los pobres del mundo. En su informe de 2005, el PNUD reporta a Islandia con el mayor IDG, las mujeres viven 3.2 años más, la educación es igual para mujeres y hombres y el ingreso de los hombres es superior 1.4 veces; en contraste, Sierra Leona tiene el menor IDG, la diferencia en la esperanza de vida también es de 3.2 años, la población alfabeta masculina casi duplica a la femenina y el ingreso masculino es 2.2 veces superior. Ni siquiera el país más avanzado ha logrado la igualdad de género. La región noreste de México presenta una desigualdad no significativa, pero aun así hay una desigualdad importante en el ingreso que debe subsanarse, además da cuenta de que el IDG no señala del todo las desigualdades injustas entre hombres y mujeres. 
Las diferencias en la región entre hombres y mujeres se manifiestan en la salud y el ingreso. Las mujeres de Nuevo León vivían en promedio 3.82 años más que los hombres, en Tamaulipas lo hacían en 4.31 años; en ese año el promedio de ingreso percibido por un hombre en Nuevo León era 2.24 veces superior al promedio femenino y en Tamaulipas era 2.05 veces (cuadro 5). En ambos casos la diferencia es seria y requiere atención, porque aunque se compensan, como se puede ver más adelante, son opciones que hombres y mujeres desearían tener, es decir, las mujeres participar proporcionalmente del mismo modo que los hombres en la economía y los hombres vivir tanto como las mujeres (cuadro 5).

\section{Cuadro 5. Valores del idhp y sus componentes según el género en la región noreste, 1995-2000-2005}

\begin{tabular}{|c|c|c|c|c|c|c|}
\hline & Años & $\begin{array}{c}\text { Esperanza } \\
\text { de vida* }\end{array}$ & InMat & InAlf & $\begin{array}{c}\text { PIB per } \\
\text { cápita** }\end{array}$ & IDHP \\
\hline \multirow{5}{*}{ Nuevo León } & 1995 & 76.27 & 0.9345 & 0.9558 & $5,229.29$ & 0.7565 \\
\cline { 2 - 7 } & 2000 & 77.12 & 0.9309 & 0.9613 & $8,295.20$ & 0.8087 \\
\cline { 2 - 8 } & 2005 & 77.8 & 0.9060 & 0.9655 & $10,171.07$ & 0.8375 \\
\hline \multirow{3}{*}{ Tamaulipas } & 1995 & 76.37 & 0.8967 & 0.9320 & $3,441.17$ & 0.7612 \\
\cline { 2 - 8 } & 2000 & 77.13 & 0.9007 & 0.9418 & $5,370.69$ & 0.7928 \\
\cline { 2 - 8 } & 2005 & 77.91 & 0.9075 & 0.9488 & $6,760.87$ & 0.8118 \\
\hline \multirow{3}{*}{ Nuevo León } & 2000 & 73.29 & 0.9232 & 0.9704 & $23,572.99$ & 0.9230 \\
\cline { 2 - 8 } & 2005 & 73.98 & 0.8986 & 0.9718 & $22,740.46$ & 0.8474 \\
\cline { 2 - 8 } Tamaulipas & 1995 & 72.39 & 0.9281 & 0.9656 & $19,467.17$ & 0.9042 \\
\cline { 2 - 8 } & 2000 & 72.93 & 0.8875 & 0.9545 & $14,241.10$ & 0.8251 \\
\cline { 2 - 8 } & 2005 & 73.60 & 0.8950 & 0.9574 & $13,866.59$ & 0.8281 \\
\hline
\end{tabular}

* Años.

** Dólares PPA.

Fuente: Cálculos propios con datos del Inegi, II Conteo 2005, XII Censo 2000 y I Conteo 1995, Banco de Información Económica (віE) у Sistema Municipal de Base de Datos (Simbad). 
En 1995 había diferencias significativas en cuanto a género en la región noreste al igual que en el país. La discrepancia en Nuevo León era de $1.67 \%$ y en Tamaulipas de $1.60 \%$, siendo Villaldama, NLN, el único municipio que no presentó desigualdad significativa de género. En el año 2000, Nuevo León se mantenía con diferencia significativa, mientras que Tamaulipas la había eliminado. Durante 2005, el estado del país que presentó menor desigualdad de género fue Yucatán, con una discrepancia de $0.37 \%$; Tamaulipas tuvo la décimocuarta posición con una discrepancia equivalente a $0.54 \%$, Nuevo León ocupó la décimonovena posición con $0.65 \%$ como diferencia, mientras que el estado que mostró mayor diferencia entre género fue Tabasco (discrepancia de $0.86 \%$ ). En ambos estados se redujo la desigualdad a más de la mitad, lo que indica que las políticas de apoyo para equidad de género, al menos en cuanto a las opciones que tienen unas y otros, han funcionado. En este mismo año, en la República Mexicana ningún estado mostró diferencia significativa de género (cuadro 5).

En la región noreste no hay municipios con desigualdad significativa de género, los municipios de mayor adelanto en la equidad de género pertenecen a Tamaulipas, los cuales son los avances más notorios de la región (cuadro 6).

Municipios de la región noreste con más avance en la reducción de la desigualdad de género en el periodo 1995-2000-2005

\begin{tabular}{|l|c|c|c|c|}
\hline \multicolumn{1}{|c|}{ Municipio } & $\begin{array}{c}\text { Diferencia } \\
1995\end{array}$ & $\begin{array}{c}\text { Diferencia } \\
2000\end{array}$ & $\begin{array}{c}\text { Diferencia } \\
2005\end{array}$ & $\begin{array}{c}\text { Reducción } \\
1995-2005\end{array}$ \\
\hline Casas (TAM) & 6.99 & 2.43 & 1.40 & 5.59 \\
\hline Cruillas (TAM) & 6.63 & 2.16 & 0.61 & 6.02 \\
\hline Méndez (TAM) & 8.80 & 2.00 & 0.54 & 8.26 \\
\hline Nuevo Morelos (TAM) & 5.60 & 1.71 & 0.73 & 4.87 \\
\hline San Nicolás (TAM) & 9.33 & 5.31 & 3.23 & 6.10 \\
\hline
\end{tabular}

Fuente: Cálculos propios con datos del INEGI, II Conteo 2005, XII Censo 2000 y I Conteo 1995, Banco de Información Económica (вік) у Sistema Municipal de Base de Datos (Simbad). 
Los municipios con menor discrepancia son: Mainero, TAM, Hualahuise, NLn, Tula, TAM, Jaumave, TAM, y Agualeguas, NLn, por mencionar algunos, cuya diferencia entre género fue de casi cero; y las más notorias se encuentran en Los Aldamas, NLn, Iturbide, NLn, Burgos, TAM, Doctor Cross, NLn, y San Nicolás, TAM, todas ellas menores al 0.5 por ciento y desfavorables para las mujeres.

\section{Variación y equidad}

En la República Mexicana, el estado de Baja California fue la entidad más equitativa, de acuerdo con valor del InQIDHP, esto significa que la mayoría de sus habitantes se encuentra en condiciones semejantes de desarrollo humano, es decir, sus municipios tienen niveles de desarrollo humano similares (IDHP alrededor de 0.8). En contraste, el estado de Oaxaca es la entidad donde prevalecía una situación de mayor inequidad, lo cual se manifiesta en una alta variación para los valores del IDHP municipales.

Se observa que a mayor pendiente de las curvas, la equidad entre municipios es mayor (gráfica 1). Tamaulipas presenta un índice de inequidad en el IDHP de 0.14156 y Nuevo León de 0.16143 , ocupando el sexto y décimotercer lugar nacional, respectivamente. Comparados con Baja California (0.11311), podemos considerarlas como entidades relativamente equitativas; los estados con mayor desigualdad son Chiapas (0.33869) y Oaxaca (0.37339), ambos con los índices más altos en la República y los que concentran mayor población indígena, al igual que Yucatán (0.19001).

$\mathrm{Al}$ interior de los estados de la región, en Nuevo León el municipio con mejor IDHP es San Pedro Garza García (0.8823), mientras que el de mayor rezago es Mier y Noriega (0.7760), una diferencia de 0.1063 (13.69\%); las posiciones respectivas en Tamaulipas están en Victoria $(0.8410)$ y Bustamante (0.7533) con diferencia de 0.0877 (11.64\%). Según los números que se observen será la magnitud de la inequidad que se aprecie: como valores absolutos se ven diferencias muy pequeñas por la escala en la que se mide el índice, como porcentajes, tales diferencias toman significancia, y si se establecieran en términos de las opciones que deja de haber para las personas en los municipios más rezagados, serían intolerables para muchas personas. ¿Qué representa en términos de opciones de salud, educación 
y recursos para una vida digna un rezago de $10 \%$ en el IDHP? Si se piensa que poco, sería incomprensible que los gobiernos no erradicaran el rezago; de otro modo, seguramente debe significar mucho, por tanto, las opciones de vida de las personas en la región, a pesar de ser altas respecto de las del estado más rezagado, están significativamente diferenciadas entre los municipios, induciendo inequidades en una escala geográfica menor.

\section{Curvas de calidad}

La capacidad para transformar el ingreso en desarrollo dentro de los estados componentes de la región está cerca de la curva promedio, la cual se encuentra entre las calidades 16 y 17. En 2005, Nuevo León obtuvo la calidad 18, que es igual a la nacional, y Tamaulipas presentó la calidad 16 avanzando una posición desde el 2000; Tlaxcala tuvo la calidad 7, por lo que se ubica como la mejor reportada para los estados dentro de la

Gráfica 1. Variación e inequidad en los estados de la región noreste y los extremos del país, 2005

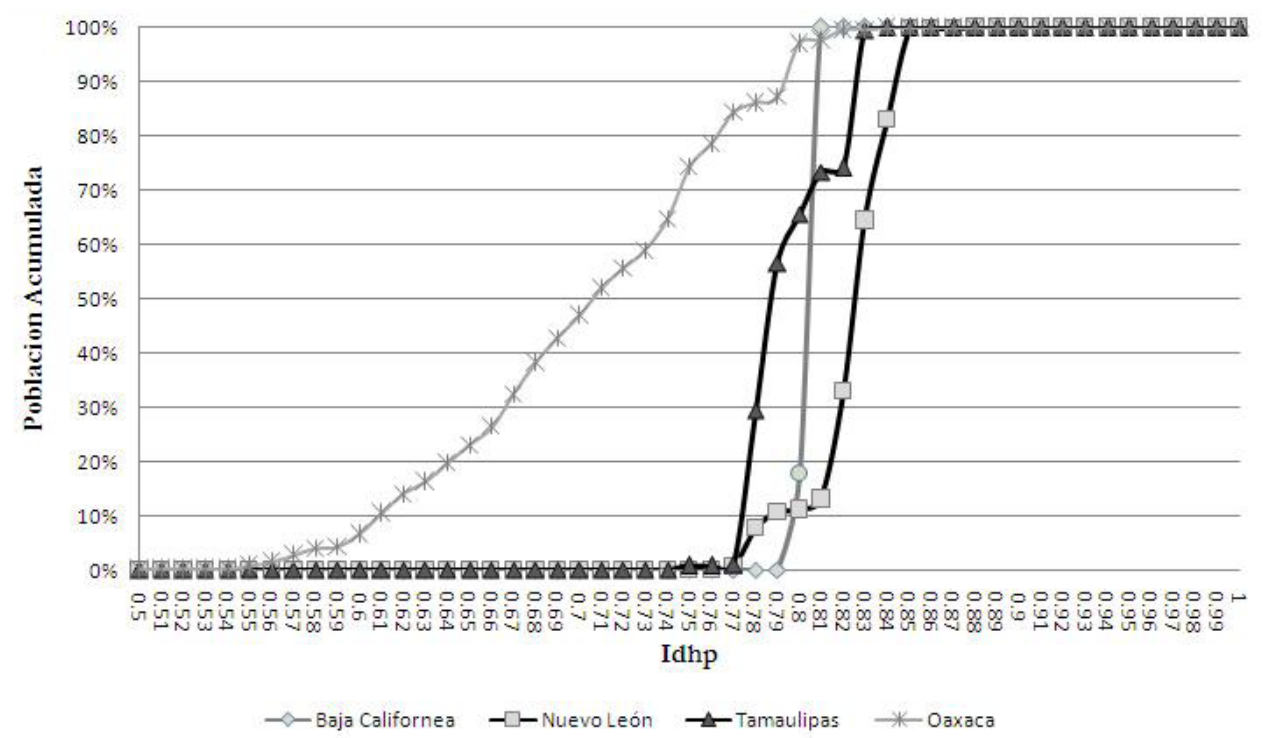

Fuente: Elaboración propia con datos del inegi, Conteo 2005, Censo 2000, Banco de Información Económica (віE) y Sistema Municipal de Base de Datos (Simbad). 
República, seguido por Nayarit (calidad 10), Colima, Estado de México y Zacatecas (calidad 11); las menos eficientes se presentaron en Chihuahua, Guerrero (ambas con calidad 21) y Campeche (calidad 23).

La calidad municipal ha ido cambiando en la región: en 1995, $25 \mathrm{mu}-$ nicipios estaban por encima de la curva guía y representaban $37.67 \%$ de la población; 58 municipios se encontraban debajo de la curva $(59.57 \%$ de los habitantes) y el resto estaba sobre la curva. Durante el año 2000 la mayoría de la población $(63.67 \%)$ tenía ineficiencia para trasformar los ingresos en servicios, en el 2005 comenzaron a mejorar la calidad; $16 \mathrm{mu}$ nicipios que representan $21.32 \%$ de la población estaban por encima de la curva guía; $50.58 \%$ repartido en 25 municipios, estaba sobre la curva y el resto con calidad deficiente. Tamaulipas mejoró bastante en el InSer, lo que le ayudó a la eficiencia de recursos en el estado (cuadro 7).

\section{Cuadro 7. Comparación de la calidad por número de municipios y población en el periodo 1995-2000-2005.}

\begin{tabular}{|c|c|c|c|c|c|}
\hline & \multicolumn{3}{|c|}{ Nuevo León } & Tamaulipas \\
\hline \multirow{3}{*}{1995} & $\begin{array}{c}\text { Curva de } \\
\text { calidad }\end{array}$ & $\begin{array}{c}\text { Número de } \\
\text { municipio }\end{array}$ & $\begin{array}{c}\text { Población } \\
(\%)\end{array}$ & $\begin{array}{c}\text { Número de } \\
\text { municipio }\end{array}$ & $\begin{array}{c}\text { Población } \\
(\%)\end{array}$ \\
\hline & Arriba & 17 & 54.17 & 8 & 14.48 \\
\cline { 2 - 7 } & Sobre & 8 & 3.20 & 3 & 2.14 \\
\cline { 2 - 7 } & Bajo & 26 & 42.63 & 32 & 83.38 \\
\hline \multirow{3}{*}{2000} & Arriba & 7 & 10.92 & 9 & 9.54 \\
\cline { 2 - 7 } & Sobre & 10 & 37.47 & 5 & 9.98 \\
\cline { 2 - 7 } & Bajo & 34 & 51.60 & 29 & 80.48 \\
\hline \multirow{3}{*}{2005} & Arriba & 8 & 10.30 & 8 & 36.61 \\
\cline { 2 - 6 } & Sobre & 17 & 52.71 & 8 & 47.61 \\
\cline { 2 - 6 } & Bajo & 26 & 36.98 & 27 & 15.78 \\
\hline
\end{tabular}

Fuente: Cálculos propios con datos del INEGI, II Conteo 2005, XII Censo 2000 y I Conteo 1995, Banco de Información Económica (вIE) y Sistema Municipal de Base de Datos (Simbad). 
Nuevo León tiene un ingreso de 10041.79 dólares PPA, el segundo mejor de la República; Tamaulipas ocupa el noveno lugar por su ingreso, el cual es de 6674.97 dólares PpA. Ambas entidades tienen un nivel de desarrollo alto en sus índices e indicadores, con excepción en la esperanza de vida, la cual se encuentra en un nivel de desarrollo medio. Ingreso más alto en Nuevo León pero semejantes niveles de desarrollo hacen que la calidad de este estado sea menor a la de Tamaulipas. Se puede ver que la calidad de los municipios no está muy lejos de la curva guía o promedio (gráfica 2).

\section{Gráfica 2. Curvas de calidad para la región noreste}

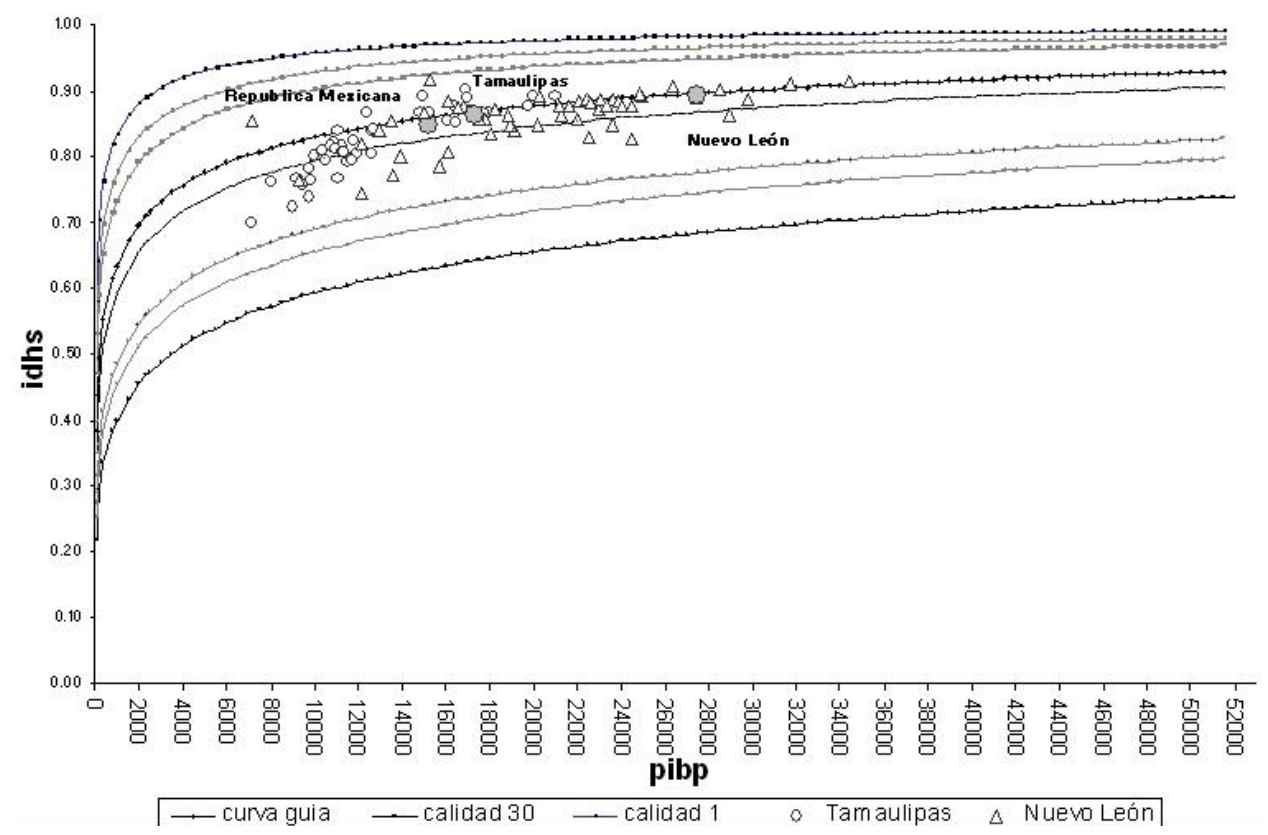

Fuente: Elaboración propia con datos del Inegi, Conteo 2005, Censo 2000, Banco de Información Económica (вІе) у Sistema Municipal de Base de Datos (Simbad). 


\section{Conclusiones}

Como se observó en el cuerpo del trabajo, la situación del país respecto de los países más desarrollados muestra un rezago importante, de modo que muchas de las condiciones favorables al interior de México, como las mostradas por la región noreste, deben considerarlo.

La región noreste tiene un Índice de Desarrollo alto (opciones mínimas para el desarrollo de las capacidades de sus habitantes), situación favorecida, inicialmente, por su inserción en la industria y el comercio y, actualmente, por la vecindad con Estados Unidos. No obstante, los estados que la componen muestran diferencias importantes en su desarrollo.

Es notorio el adelanto de Nuevo León respecto de Tamaulipas, de modo que aún entre los dos estados vecinos y en condiciones favorables de vecindad con Estados Unidos se notan disparidades significativas. En la evolución de los indicadores se observa cómo Tamaulipas viene remontando el rezago, quedando a la zaga en el 2005 sólo en el PIвp y el índice de drenaje. Esta tendencia a un desarrollo equiparable fortalecerá a la región social y económicamente.

Los esfuerzos en Tamaulipas para superar el rezago en los servicios son muy notorios, aun en el drenaje, donde en México se tienen rezagos ancestrales. Sólo en los últimos sexenios se ha visto una política para resarcir el abandono de los servicios por muchos años. Una condición digna de vida, como se ha favorecido, permitirá el concurso y la participación de más personas en la esfera social y económica de la región. Así, se combatirá la pobreza humana y de carencia de ingreso.

El municipio que se mantiene a la cabeza en la región noreste es San Pedro de los Garza García, nln, conocido como el Beverly Hills mexicano; se distingue por estar habitado por los empresarios más emblemáticos del país, las clases políticas del estado y los ejecutivos de empresas transnacionales. En contraparte al buen desarrollo promedio de la región, se tiene a Bustamante, Michihuana y San Nicolás, municipios de Tamaulipas, donde las principales actividades son la agricultura, ganadería y minería; casi una cuarta parte de sus habitantes es analfabeta y poco más de $60 \%$ de la población municipal son personas económicamente inactivas. Esta polaridad afecta la armonía del tejido social y alienta diferencias en la concepción de 
un bienestar común ya que las personas son vistas como medios y no fin de los procesos de desarrollo.

Dentro de la región, las actividades económicas presentes en los municipios con mejor desarrollo son principalmente las de los sectores secundario y terciario, observándose que los municipios con menor desarrollo se dedican básicamente al sector primario y la población regional que los habita es menor a 1\%. Hay evidentemente una asociación entre el desarrollo y las actividades económicas, así como de éstas y los tamaños poblacionales, determinando círculos viciosos que construyen el rezago y la marginación o círculos virtuosos que mantienen un liderazgo permanente. De este modo, la región ofrece contrastes que deterioran la equidad del desarrollo y abonan inconformidades sociales que la detienen, las cuales urgen una política social que considere las diferencias y evite la reproducción de tales círculos, preocupándose por el desarrollo directo de las personas y no como consecuencia de procesos ligados a otros fines.

Los municipios con los valores más bajos se repiten en casi todos los factores, señalando lo pernicioso del rezago cuando el desarrollo obedece a metas ajenas a los seres mismos. Lo mismo pasa a escala estatal con los estados de Chiapas, Oaxaca y Guerrero. Esta condición que se observa en todas las regiones que ofrecen polaridades en su desarrollo debería indicar a los estados la necesidad de políticas públicas especiales para romper las inercias que no permiten un desarrollo equitativo. Ensayar las mismas políticas entre territorios desiguales es reproducir la desigualdad. Políticas que asignen recursos de manera diferenciada, otorgando más a los territorios con menor desarrollo bajo una vigilancia especial, están obligadas si no se quiere seguir edificando la desigualdad.

La escolaridad en Nuevo León tiene uno de los mejores índices estatales, pero presenta un retroceso muy importante en el índice de matriculación. De 2000 a 2005 más de dos niños de cada 100 entre seis y 14 años se agregaron a los que no sabían leer y escribir (INEGI, 2009), señalando una omisión importante del Estado que puede minar en el futuro el avance que ha tenido en el desarrollo de sus habitantes. Nuevo León es uno de los estados más desarrollados en el área económica, su actividad de empresas e industrias y la creciente infraestructura lo han colocado en la competencia a escala internacional. Desatender la educación en la situación econó- 
mica en que se encuentra puede ser lamentable por la necesidad que tiene de recursos humanos mejor formados.

Dentro de la región la desigualdad de género no es significativa, el InAlf femenino es inferior al masculino por menos de $1 \%$, mientras que en salud e ingreso las desigualdades se compensan, los hombres producen más bienes y servicios y las mujeres viven aproximadamente cuatro años más en promedio. Mucho del adelanto de las mujeres en la década estudiada se debe a su avance en el PIBP, el cual creció muy por encima del de los hombres y en algunos estados fuera de la región se mantuvo sin cambio o disminuyó. Esta situación, de continuar, augura una inequidad para los hombres, porque mientras es fácil la transferencia de trabajos de los hombres a las mujeres, no lo es en el aspecto de salud, donde los hombres tienen rezagos. A ello se debe la reducción tan drástica que se ha tenido en la inequidad de género desfavorable, hasta hoy, en las mujeres.

En cuanto a la forma en que se distribuye el desarrollo humano en los estados de la región, si se mide en la escala estatal resulta equitativa; sin embargo, al interior de los estados los municipios ofrecen grandes contrastes, pues hay pequeñas poblaciones en los extremos, principalmente en Nuevo León, creando una polaridad que exhibe los problemas que las personas tienen para acceder de forma equitativa a los diferentes servicios para su pleno desarrollo.

La mejor calidad en la región es Tamaulipas, ligeramente superior a la de Nuevo León, pues teniendo menos recursos que éste, ha alcanzado un desarrollo mayor al promedio esperado para el país. No obstante, al ser uno de los principales productores de electricidad en el país se esperaría que su InEle fuera uno de los mejores. La inversión en drenaje fue clave para elevar su calidad. El IDHs de Nuevo León es alto y se ha mantenido de esta manera pero sin avances notorios; en general ha ido creciendo a la par de la población, dando prioridad a los sitios más poblados. Lo anterior puede señalar un foco rojo para Nuevo León, cuyo modelo de desarrollo acusa mayor similitud con el económico que el humano.

Los índices analizados nos permiten ver que, en general, la región noreste presenta condiciones para el desarrollo de sus habitantes. Aún faltan oportunidades de acceso oportuno a la educación, salud y vivienda digna. De acuerdo con el análisis de cada uno de los indicadores, sus resultados no sólo son producto del funcionamiento de un solo factor, sino también 
del efecto de la acción conjunta de todos los factores implicados en el desarrollo humano, como la dispersión demográfica, la falta de comunicaciones, la inequidad en la distribución del ingreso, etcétera. De este modo, es apreciable en la región el adelanto que tuvo Nuevo León en el siglo xix y su influencia sobre Tamaulipas, visible ahora en sus mejores índices de desarrollo humano.

En los índices considerados no se tiene ninguna medición de la violencia y su impacto en la salud tanto física como psíquica, por lo que no se ve reflejado en las condiciones de bienestar de la población; aspecto importante en la región asociado al narcotráfico, aun cuando no sea la zona más crítica de México.

La solución de los problemas de un componente de desarrollo debe plantearse a partir de verdaderas políticas integrales dirigidas a las personas, y debe incluir la dimensión territorial, atendiendo a la heterogeneidad geográfica, económica, demográfica, cultural, etcétera, de un municipio, estado, país o cualquier espacio habitado por seres humanos (Zamudio, 2004).

\section{Bibliografía}

Bassols, B. A. (1967), La división económica regional de México, México, Universidad Nacional Autónoma de México.

— (1999), "Investigaciones urbanas y regionales de México: ¿para conocer o transformar una realidad?", en Humberto Muñoz (coord.), México. La sociedad mexicana frente al tercer milenio, México, Miguel Ángel Porrúa, Coordinación de Humanidades.

Demyc (2007), Departamento de Estadística, Matemática y Computo. Obtenida el 12 de Agosto de 2007, http://www.chapingo.mx/dicifo/demyc/idh/new/bases new/memo/memoria.html

INEGI (1999), "Anuario estadístico. Los municipios de Nuevo León y Tamaulipas", en www.inegi.gob.mx. Fecha de consulta: 30 de abril de 2008.

(2005), Conteo de Población y Vivienda, X Censo General de Población y Vivienda, México, Banco de Información Económica, Sistema Municipal de Bases de Datos, Instituto Nacional de Estadística Geográfica e Informática, en www. inegi.gob.mx. Fecha de consulta: febrero de 2009.

(2009), Cuéntame, Informacion porentidad, en < http://cuentame.inegi.gob.mx/ monografias/informacion/nl/poblacion/educacion $\cdot \operatorname{aspx}$ ?tema $=$ me\&e $=19>$. Fecha de consulta: 17 de junio de 2009. 
Jagaar, A. M. (1983), Feminist Policies and Human Nature, Totowa, Nueva Jersey, Rowmann and Alaheld.

Kaldor, N. (1961), The Theory of Capital. "Capital Accumulation and Economic Growth", Nueva York, St Martin's Press.

McKinnon, C. (1987), Feminism Unmodified: Discourses of Life and Law, USA, Cambridge, Massachusetts, Harvard University Press.

Martínez, C. J. C. (2001), "Los mercados de factores", en La economía de mercado, virtudes e inconvenientes, en <http://www.eumed.net/cursecon/7/Lorenz-Gini. htm >. Fecha de consulta: 20 de marzo de 2007.

Pettinato, S. (2002), "A Conceptual Primer on the Currents and Trends in Inequality", Journal of Human Development, Estados Unidos.

PNUD (1990), Informe sobre desarrollo humano 1990, Colombia, Programa de las Naciones Unidas para el Desarrollo, Tercer Mundo Editores.

- (1995), Informe sobre desarrollo humano 1995, México, Programa de las Naciones Unidas para el Desarrollo, Harla.

(2005), Informe sobre desarrollo humano 2005, México, Programa de las Naciones Unidas para el Desarrollo, Ediciones Mundi-Prensa.

(2007-2008), Informe sobre desarrollo Humano 2007-2008, México, Programa de las Naciones Unidas para el Desarrollo, Ediciones Mundi-Prensa.

— (2011), Human Develpoment Report: Sustainability and Equity: A Better Future for $A l l$, Nueva York, United Nations Development Programme, Palgrave Mcmillan. (2012), "El Índice de Desarrollo Humano en México: cambios metodológicos e información para las entidades federativas", México, Offset Santiago, en http://www.undp.org.mx/ImG/pdf/Boletin_IDH.pdf. Fecha de consulta: 9 de diciembre de 2012.

Ramírez, A. (1999), "Índice de Desarrollo Humano en el estado de Guanajuato", Revista del Centro de Desarrollo Humano de Guanajuato, núm. 3.

Ranis, Stewart y Alejandro Ramírez (2000), "Economic Growth and Human Development", World Development, vol. 28, núm. 2, Londres, Elsevier.

Sen, A. (1980), Equality of What?, Nueva York y Cambridge, University of Utah Press, Cambridge University Press.

(1987), The Standard of Living, Nueva York, The Press Syndicate of the University of Cambridge.

— (1994), "Sustaineble Human Development: Concepts and Priorities", undP, en $<$ http://hdr.undp.org/docs/publications/ocational_papers/oc8a.htm > . Fecha de consulta: 1 de abril de 2010.

Simbad (2003), México, Sistema Municipal de Base de Datos, en <http://sc.inegi. gob.mx/simbad/index.jsp?c $=125>$. Fecha de consulta: 20 enero de 2008. (2005), México, Sistema Municipal de Base de Datos, en <http://sc.inegi. gob.mx/simbad/index.jsp?c=125>. Fecha de consulta: 15 de febrero de 2008. 
Zamudio, S. (2004), Segundo Informe sobre Desarrollo Humano de los Estados de Tamaulipas y Veracruz: Análisis Comparativo 1995-2000, Chapingo, México, Universidad Autónoma Chapingo. Departamento de Estadística, Matemática y Cómputo.

, A. A. Corona y M. González (2007), "El desarrollo humano en el Estado de México y el Distrito Federal, 1995-2000: una lejana vecindad”, Economía, Sociedad y Territorio, vol. viI, núm. 25, en <http://redalyc.uaemex.mx/redalyc/ src/inicio/ArtPdfRed.jsp?iCve $=11102504>$. Fecha de consulta: 13 de marzo de 2010.

Artículo recibido en enero de 2012. Segunda versión aprobada el 31 de enero de 2013. 